\title{
Design of Athlete's Running Information Capture System in Space-Time Domain Based on Virtual Reality
}

\author{
Chun Zhu (1) \\ Wuhu Institute of Technology, Wuhu 241006, China \\ Correspondence should be addressed to Chun Zhu; zhunchun@whit.ah.cn
}

Received 10 November 2021; Revised 29 November 2021; Accepted 3 December 2021; Published 3 February 2022

Academic Editor: Baiyuan Ding

Copyright ( 92022 Chun Zhu. This is an open access article distributed under the Creative Commons Attribution License, which permits unrestricted use, distribution, and reproduction in any medium, provided the original work is properly cited.

\begin{abstract}
In order to improve the training effect of athletes, aiming at the problems of inaccurate information capture results, poor real-time performance of sports information capture, and inability to effectively suppress noise interference in traditional methods, an athlete space-time running information capture system based on virtual reality is designed. Establish the athlete's human skeleton, obtain the athlete's sports joint points, design the overall architecture of the athlete's space-time running information capture system, and realize the whole link design of sports information capture through RF chip, infrared camera, sports data acquisition module, data transmission module, and human-computer interaction module. Based on virtual reality technology, a virtual reality environment is built to obtain the characteristic parameters of athletes' sports posture in space-time domain, and the median filtering algorithm is used to filter the original signal to eliminate the impact of noise signal on athletes' sports information capture. Finally, the activity of the motion region is detected, and the motion information is captured combined with the Gaussian mixture model. The experimental results show that the system designed in this paper has high accuracy and anti-interference and can realize the real-time capture of motion information.
\end{abstract}

\section{Introduction}

According to the training characteristics of athletes, an athlete sports information collection system is designed, which can collect relevant data according to the athlete's training data by formulating a reliable communication protocol, using special instruments and transmitting them to the computer through IC card, display various data parameters, and automatically generate accurate training data from the original data by software [1-3]. Through the application of the system, it can provide auxiliary decisionmaking means for athletes to exercise scientifically and reasonably. Nowadays, mature human motion capture systems in the market include optical, electromagnetic, and ultrasonic [4-6]. These systems have disadvantages in practical application. They have high requirements for the site and inconvenient installation. The most important thing is that the price is expensive, which is difficult for most athletes to afford, making it difficult for athletes' motion capture systems to be popularized [7].
In view of the above problems, relevant scholars are committed to studying an effective athlete sports information capture system. Reference [8] proposes a human knee motion capture system based on MATLAB. MATLAB software is used to analyze and process the motion of human knee. Firstly, a program is written to process the coordinated data of human lower limb marker points measured by motion capture and interpolate them. Secondly, according to the obtained coordinate data of bone marker points in the process of human lower limb movement, the local coordinate system of human femur and tibia is established by using bone marker points. Finally, through coordinate transformation, the flexion, extension, adduction, abduction, and internal and external rotation of knee femoral tibial joint in human lower limb movement are calculated. This method can quickly process the motion data of human knee joint and obtain the kinematic characteristics of femoral tibial joint of human knee joint, which provides a reference for athlete training. Reference [9] designed a human motion trajectory tracking system based on three-frame difference method. 
The system conducted in-depth research on the underwater environment, set up initialization layer, motion detection layer, and human tracking layer in the hardware architecture, and constructed three-dimensional human model, motion feature extraction module, and motion feature segmentation module so as to determine the boundary features and gray features of human body, contour features, and skin color features. The tracking trajectory of the system is highly similar to the actual running trajectory. Reference [10] proposes a three-dimensional human posture tracking method based on dual Kinect sensors, which represents the human motion posture by the method of human joint degree of freedom vector, uses the traceless Kalman filter method to track the human posture, and constructs a set of human posture tracking system based on dual Kinect sensors. Compared with the traditional human motion capture system, the system can realize three-dimensional human posture tracking and reflect the special properties of the motion process under complex actions.

Although the above systems have effectively solved the shortcomings of traditional methods, some new problems have been found in the application, such as the inaccurate information capture results of some joints in the motion state, the poor real-time performance of motion information capture, and the inability to effectively suppress noise interference. Therefore, this paper designs a running information capture system based on virtual reality. With the help of computers, virtual reality can bring people realistic feelings such as seeing and listening, and with the help of specific instruments and equipment, people can interact in the virtual environment created by computer.

\section{Hardware Design of Athletes' Running Information Capture System in Space- Time Domain}

2.1. Establishment of Athletes' Human Skeleton. In order to accurately describe the movement state of athletes, it is necessary to establish a skeleton model for the human body. As shown in Figure 1, some human joint nodes are given. There are 21 joint points in the human body, including 38 joint degrees of freedom and 6 degrees of freedom. The motion of the human body can be regarded as the motion of these joint points. When capturing the motion of these joint points, the motion of the skeleton can be determined.

It can be seen from the skeleton model that the rotational motion degrees of freedom of each marked point are not the same. According to the rotational motion degrees of freedom of the marked points, they can be divided into four categories:

(1) Fixed, nonrotatable marker points, can be used to measure the displacement of the whole skeleton model in space, such as hips.

(2) Hinge shaped, the marking point connects two parts, with a degree of freedom of rotation, such as the marking point at the elbow.

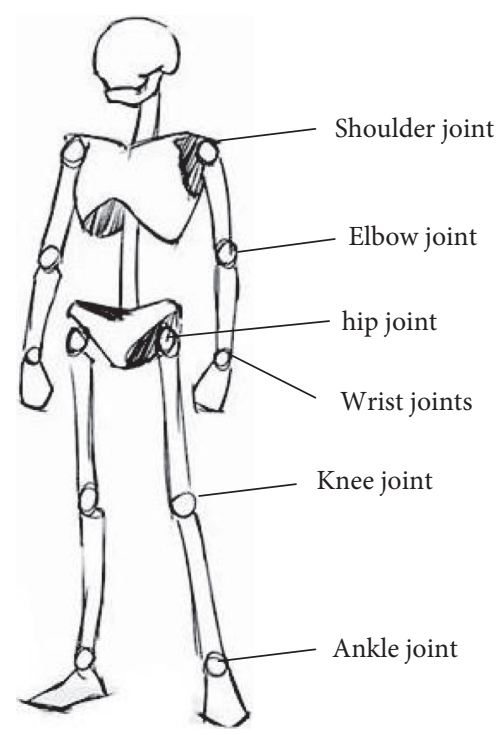

FIgURE 1: Athletes' skeleton model.

(3) Spherical, the marking point has three rotational degrees of freedom, such as the marking point of shoulder joint.

(4) Generally, this marker point has two rotational degrees of freedom, such as ankle marker point.

Such classification is helpful to reduce the time complexity of the algorithm and improve the accuracy of tracking in motion capture. In the specific operation process, too many marked points will increase the amount of calculation, and too few marked points will make the action impossible to be accurately restored, resulting in abnormal action. Therefore, the number of marked points should be determined according to the task to be completed.

\subsection{Overall System Architecture and Hardware Module Design}

2.2.1. Overall System Architecture. In the overall design of the athlete's time-space running information capture system, the signal input can be electrical signals, physical signals, and so on. In the forward channel, various signals are converted into electrical signals through sensors, and then the signals are converted into digital signals through ADC. PCI, VXI, ISA buses, and so on are inserted into the computer to work to realize the simulation of the athlete's running posture. Combined with the skeleton model of the human body, the sensor nodes are arranged to realize the full information perception of the athlete's movement posture.

This article comprehensively considers system technical indicators, system performance, development difficulty and development cost, and so on, chooses 5409A as the main processor of the system, and chooses PCI bus and PCI9054 bridge chip as the system data communication interface. The overall design model structure of the system is as shown in Figure 2.

Since the athlete's motion capture system requires a total of 21 joint data information, 17 sensors must be used to collect the attitude information of the carrier. These 21 sensors are 


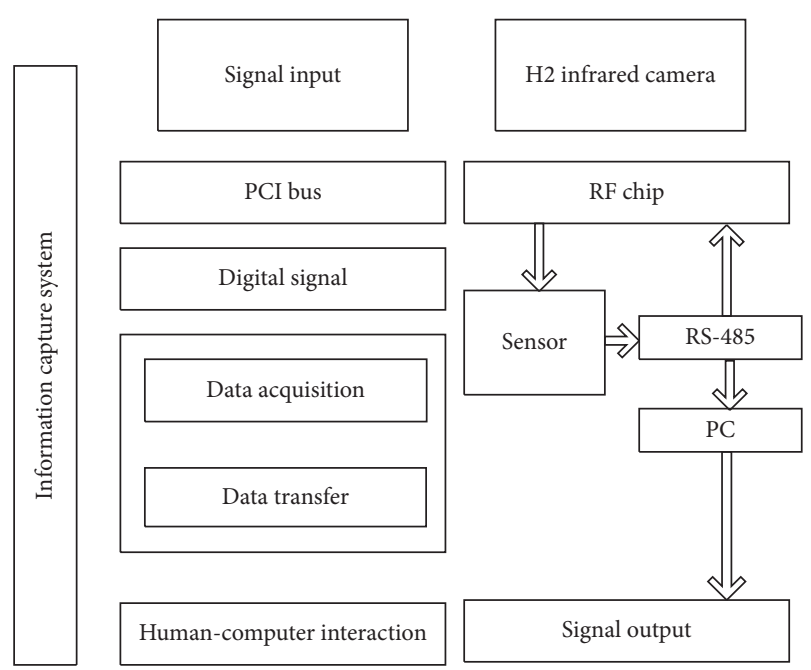

FIGURE 2: The overall architecture diagram of the athlete's running information capture system in the temporal and spatial domains.

divided into 3 channels, and each channel uses an RS- 485 bus to connect 7 sensors and a signal catcher is then connected to the transmitter through the signal catcher and transmitted to the receiver on the PC side through the wireless network, and then the receiver is connected to the PC.

\subsubsection{Hardware Module Design}

(1) RF Chip Selection. In order to meet the requirements for real-time changing athletes' running posture recognition, this system adopts an ultra-high-frequency RFID system. Through comparative analysis, it is found that the nRF24LE1 radio frequency chip produced by Nordic has the characteristics of ultra-low-power consumption, small size, long range, and low cost. nRF24LE1 is a highly integrated, ultra-low-power consumption, system-on-chip technology $2.4 \mathrm{GHz}$ radio frequency chip [11]. It integrates a radio frequency transceiver, an enhanced 51 FLASH high-speed microcontroller, and a wealth of peripherals and interfaces in a very small package. The radio frequency transceiver works in the ISM frequency band of $2.400 \mathrm{GHz}$ to $2.483 \mathrm{GHz}$ and adopts GFSK modulation and demodulation with strong anti-interference ability, and the transmission rate can reach $2 \mathrm{Mbps}$. The peak current provided by RX/TX is less than $14 \mathrm{~mA}$, there is a power saving mode below $\mu \mathrm{A}$, advanced power management, and the power supply range is 1.9 to $3.6 \mathrm{~V}$. nRF24LE1 truly achieves ultra-low-power consumption performance. What is more worth mentioning is that nRF24LE1 provides an Enhanced ShockBurst ${ }^{\mathrm{TM}}$ transceiver mode. In this mode, data is sent in at a low speed, but it can be transmitted at a high speed ( $2 \mathrm{Mbps}$ ), which not only saves energy, but also improves anti-interference. The embedded fast 8-bit MCU executes the traditional 8051 instruction set, but the speed is 8 times that of the traditional 8051 microcontroller. It has high execution capability and powerful functions, and high-speed data transmission through the SPI interface with the radio frequency transceiver [12].
In addition, the RFID system inevitably has to consider the signal collision problem of multiple tags entering the reading area at the same time, and the nRF24LE1 integrates the carrier monitoring function, which can accurately monitor whether the current working channel has interference. Therefore, the system chooses nRF24LE1 as the core chip, which can not only meet the requirements of low cost, low power consumption, high integration, and high performance, but also enhance the system's ability to prevent collisions and identify moving targets $[13,14]$.

(2) Infrared Camera. In order to collect high-definition images of athletes' running posture, the $\mathrm{H} 2$ model infrared camera was selected, which has a CAD infrared light bluetooth camera aimed at 60 meters. Using this camera, the photoelectric conversion main board and internal and external dual optical path correction optics can be used as compensation to reduce signal interference, so it can make the signal more stable and ensure that the measurement data are also in a stable state [15]. Choose a high-quality 2-million-pixel camera, equipped with a large-memory high-speed multimedia platform; the picture quality is clear and smooth, and it is easier to capture the athlete's movement posture. 1000 sets of data are stored and exported, which can be read directly in the machine, or exported using a USB connection to a computer [16]. The specific parameters of the $\mathrm{H} 2$ model infrared camera are shown in Table 1.

It can be seen from Table 1 that the $\mathrm{H} 2$ model infrared camera is a camera that integrates a camera, goggles, infrared camera, and heat sink. The basic principle is to use ordinary CCD black-and-white cameras to perceive the spectral characteristics of infrared light and combine infrared lamps as the "light source" for night vision imaging. The power, angle, camera configuration of infrared lamps, and infrared lenses with a certain focal length can realize rapid imaging of athletes' movement posture in a dynamic environment.

(3) Movement Data Acquisition Module. The generation and collection of motion posture data is the basis of the entire information capture system, which controls the dynamic gain code of the analog signal preprocessor and performs spectrum analysis on the signal $[17,18]$. In summary, the data acquisition model of the motion posture data capture is obtained. The workflow of the motion data acquisition model is as shown in Figure 3.

In Figure 3, sensor nodes are arranged at each joint bone node of the human body to read the posture data of the human body movement and build a human body behavior database based on the read data information.

(4) Data Transmission Module. In the above motion data acquisition module, ARM Cortex-M3 series chips are used to collect data and send it to the transmitter. The transmitter also adopts ARM chip. The difference is that the device also includes a transmitting chip with $2.4 \mathrm{G}$ wireless network, which uses NRF24L01 [19]. Through this chip, the data is sent out wirelessly. The receiver is also a processor with ARM chip and $2.4 \mathrm{G}$ wireless receiving device with NRF24L01 
TABLE 1: H2 model infrared camera parameters.

\begin{tabular}{lc}
\hline Parameter & Specification \\
\hline Measuring range & $>50 \mathrm{~m}$ \\
Precision & $\pm 2 \%$ \\
Infrared camera resolution & $256 * 192$ \\
Visible light resolution & $1920 * 1080$ \\
Size & $54 * 25 * 79 \mathrm{~mm}$ \\
Frame rate & $25 \mathrm{~Hz}$ \\
\hline
\end{tabular}

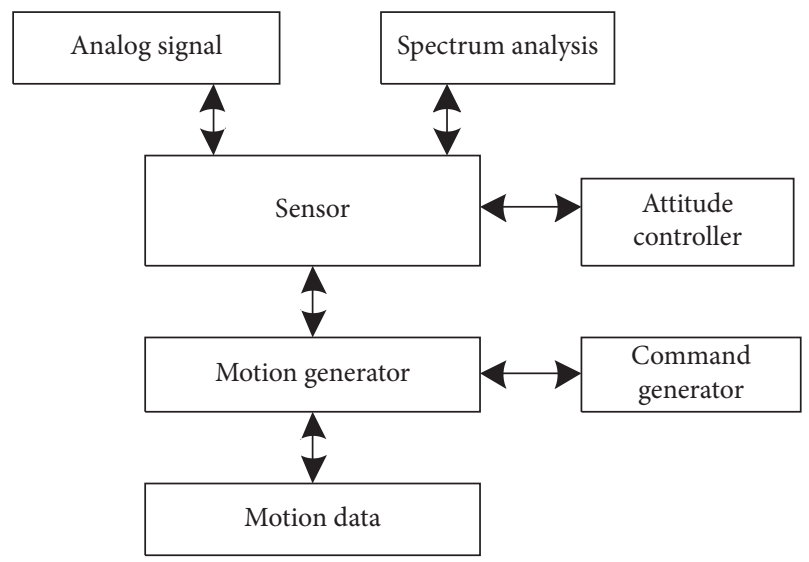

FIGURE 3: Workflow chart of the motion data acquisition module.

chip. After receiving the data from the transmitter through the receiver, it is sent to the PC through the serial port.

NRF24L01 is a single-chip wireless transceiver chip produced by NORDIC that works in the ISM frequency band of $2.4 \mathrm{GHz}-2.5 \mathrm{GHz}$. The wireless transceiver includes frequency generator, enhanced "ShockBurst" mode controller, power amplifier, crystal oscillator, modulator, and demodulator [20]. It can almost be connected to various single-chip computers. Even if the SPI interface is not available, the SPI interface can be simulated through the ordinary IO port, and finally the data transmission is completed. Its main performance parameters are as shown in Table 2.

(5) Human-Computer Interaction Module. The ROS stack of the human-computer interaction module obtains the athlete's motion information through human body gesture recognition and voice recognition and displays the information on the system's human-computer interaction interface. The remote ROS stack is responsible for solving the received posture information and, at the same time, recognizing the athlete's current posture and feeding it back to the human-computer interaction control terminal [21, 22]. The real-time data synchronization and interaction between different ROS stacks are carried out through the wireless network and displayed on the main display interface.

The ROS stack of the human-computer interaction module includes two parts: the athlete's motion gesture recognition package and the adaptive speech recognition package. The human-computer interaction module obtains the athlete's movement information through the athlete's movement posture recognition and voice recognition and at the same time receives the movement data collected from the remote movement data acquisition module and displays the
TABLE 2: Specific parameters of the data transmission module.

\begin{tabular}{lc}
\hline Parameter & Specification \\
\hline Voltage working range & $1.9 \mathrm{~V}-3.6 \mathrm{~V}$ \\
Temperature range & $-40^{\circ} \mathrm{C}-80{ }^{\circ} \mathrm{C}$ \\
Working frequency & $2.4 \mathrm{GHz}-2.525 \mathrm{GHz}$ \\
Transmit power & $-6 \mathrm{dBm},-12 \mathrm{dBm},-18 \mathrm{dBm}$ \\
Receive working current & $12.3 \mathrm{~mA}$ \\
Launch operating current & $11.3 \mathrm{~mA}$ \\
Data transfer rate & $1 \mathrm{Mbps}$ \\
Packet received length & $1-32$ bytes \\
\hline
\end{tabular}

athlete's movement posture information on the humancomputer interaction interface [23].

Through the above analysis, it can be seen that the athletes' running information capture system designed in this paper realizes the whole link design of athletes' sports information collection, transmission, and interaction through infrared camera, sports data acquisition module, data transmission module, and human-computer interaction module so as to provide reliable hardware support for athletes' sports information capture.

\section{Software Design of Running Information Capture System for Athletes in Time and Space}

3.1. Construction of Virtual Reality Environment. Virtual reality is based on computer technology as the core, combined with related science and technology, to generate a digital environment that is highly similar to a certain range of real environments in terms of sight, hearing, and touch. Users use necessary equipment to interact and interact with objects in the digital environment and can experience the feeling of adapting to the real environment. Virtual reality is created by human beings in the process of exploring and understanding nature and gradually formed a scientific method and technology for understanding nature, simulating nature, and better adapting and using nature.

The construction of the virtual reality environment is the basis of the software design of the running information capture system of the entire athlete's time and space domain, which mainly includes three-dimensional visual modeling and three-dimensional auditory modeling [24, 25]. Among them, visual modeling mainly includes geometric modeling, motion modeling, physical modeling, object behavior modeling and model segmentation, and so on [26, 27]. Auditory modeling usually just adds interactive sound responses to the activities of users and objects. The modeling cycle of the virtual reality environment is as shown in Figure 4 .

Geometric modeling is an abstract model used to describe the inherent geometric properties of an object. The content expressed includes the shape of the object and the appearance of the object. The shape of the object can be represented by points, straight lines, polygonal figures, curve or surface equations, or even images. And so on, it depends on the comprehensive consideration of storage and computing overhead. Abstractly representing the outline and 


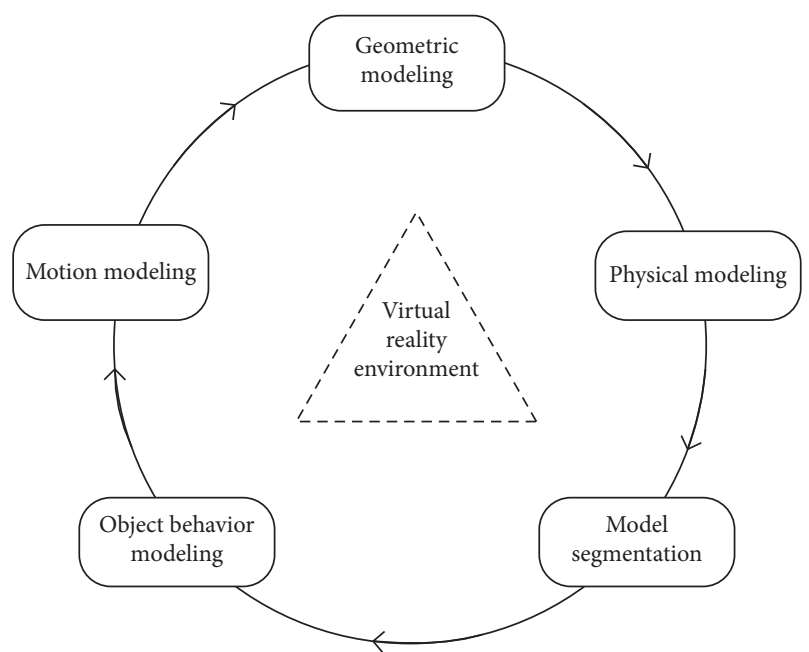

Figure 4: Virtual reality environment modeling cycle.

shape of primitives in an object is conducive to storage but needs to be recalculated when used. Specifically, the calculation time can be saved during generation, but the time and space overhead required for storage and access to the storage is relatively large. The content of the object's appearance description includes surface texture, surface light intensity, and surface color.

After setting the shape and appearance of the object, the next step is motion modeling [28]. In general, $4 \times 4$ homogeneous transformation matrix is used to describe the motion modeling so as to determine the position of the three-dimensional object in the coordinate system. The general form of homogeneous transformation matrix is

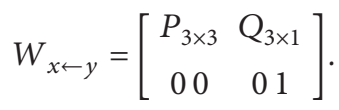

Among them, $P_{3 \times 3}$ represents the rotation submatrix of the coordinate system $x$ relative to the coordinate system $y$; $Q_{3 \times 1}$ represents the position vector of the origin of the coordinate system $x$ relative to the origin of the coordinate system $y$. The method of using homogeneous transformation matrix to orthogonalize two coordinate systems can save the amount of calculation. Rotation and translation can be defined in the same way, and they can also be combined. The inversion is relatively easy and suitable for complex modeling.

$$
\begin{aligned}
& W_{x \leftarrow y}=W_{y \leftarrow x} \\
& =\left[\begin{array}{cc}
P^{W} & P^{W} H \\
00 & 01
\end{array}\right] .
\end{aligned}
$$

When the object is scaled, it is only necessary to multiply the scale factor about the three axes of the world coordinate system in the diagonal elements of the $P_{3 \times 3}$ matrix; the rotation of the object only needs to be transformed in the rotation submatrix $P_{3 \times 3}$. time and space domain of the athlete's motion information so that real-time motion information can be obtained from the simulation image in virtual reality, and real-time motion simulation is realized. The captured motion information is simulated by the forward kinematics theory. In using virtual reality to simulate athletes' movements, each joint chain is set with at least 3 nodes, and at the same time, its own local coordinate system is established for the joint chain nodes $[29,30]$.

Set the coordinates of node $u$ in the world coordinate system to $\left(x_{u}, y_{u}\right)$, and set the coordinate position of node $v$ to $\left(x_{v}, y_{v}\right)$, and the position information of node $u$ and node $v$ is obtained as follows:

$$
\left[\begin{array}{l}
x \\
y \\
1
\end{array}\right]=\left[\begin{array}{ccc}
1 & 0 & -x_{v} \\
0 & 1 & -y_{v} \\
0 & 0 & 1
\end{array}\right]\left[\begin{array}{c}
x_{u} \\
y_{u} \\
1
\end{array}\right] .
$$

For multinode links, in fact, the conversion matrix between the local coordinate systems of $u$ to $v$ can be obtained by the mutual combination of adjacent parent nodes; namely,

$$
D_{u \longrightarrow v}=d_{u \longrightarrow u+1} \cdot H_{v \longrightarrow v+1} \cdots H_{u \longrightarrow u+n} \cdot H_{v \longrightarrow v+m},
$$

where $D_{u \longrightarrow v}$ represents the combined transformation matrix from node $u$ to node $v$. Through formula (4), all nodes in the coordinate system can be represented as a complete joint chain. Expand formula (4) and find that the coordinate position of the node can be expressed as a continuous function:

$$
G=\left(g_{1}, g_{2} \cdots g_{N}\right) .
$$

Among them, $N$ represents the number of rotation angles between nodes. Through the acquisition of the characteristic parameters of the athlete's movement posture in the time and space domain, the joint movement of the athlete in the sense of the time and space domain is determined.

3.3. Athlete Motion Signal Filtering. In order to eliminate the influence of noise signals on athletes' motion information capture, it is also necessary to preprocess the collected data. This paper uses the median filter algorithm to filter the original signal. The median filter technology has a good filtering effect on impulse noise, especially while filtering the noise, it can still retain the characteristics of the original sensor data. The comparison effect of the collected sensor's original motion signal and the median filtered motion signal is as shown in Figure 5.

The motion signal waveform after median filtering is smoother, which eliminates waveform burrs and jitter caused by noise signals and at the same time corrects samples with large deviations for further processing.

3.4. Regional Activity Detection. When using virtual reality technology to analyze the behavior of athletes, areas of intense movement can provide more discriminative 


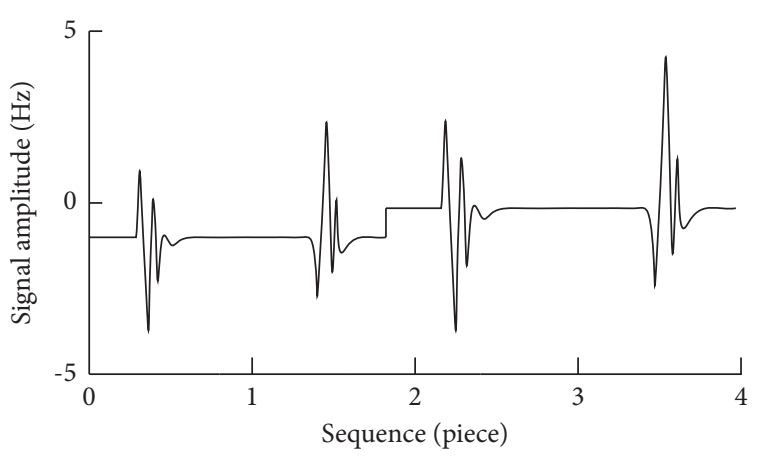

(a)

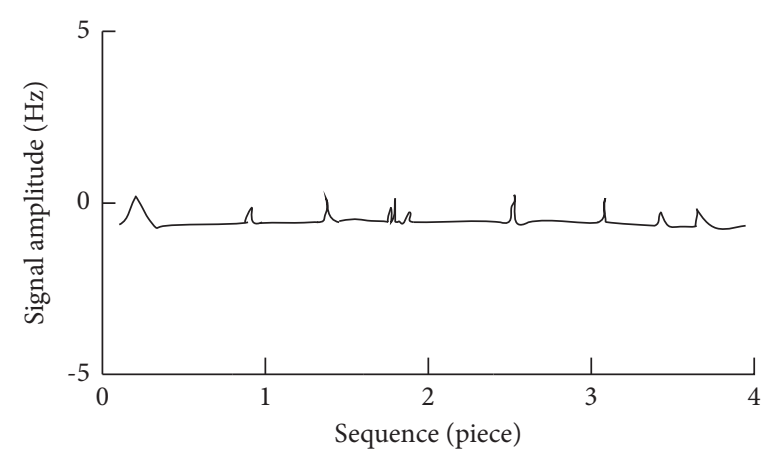

(b)

Figure 5: Comparison of motion signals before and after median filter processing.

information. Calculating the Lucas-Kanade optical flow characteristics of the area, as an energy function, can evaluate the degree of activity in the area. The calculation method is shown in the following formula:

$$
F(k)=\left[1-\frac{\left(f_{\text {max }}^{t}-f_{\text {min }}^{t}\right)}{f^{t}}\right] \exp \left(k_{u} k_{v}-\theta(k)\right) .
$$

Among them, $F(k)$ represents the optical flow vector of the $k$ th pixel in the area; $\theta(k)$ represents the weight value of the pixel in the area, usually $\theta(k)$ takes a constant; $t$ represents the number of pixels in the area; and $f^{t}$ represents the magnitude of the optical flow vector value. The calculation method is as shown as follows:

$$
f^{t}=f_{\text {best }}^{t}-r\left(f_{\max }^{t}-f_{\min }^{t}\right) \text {. }
$$

Among them, $r$ represents the energy function in the area. The larger the value, the more active the area is.

3.5. Motion Information Capture Algorithm Based on Gaussian Mixture Model. The Gaussian mixture model refers to the use of $Z$ Gaussian distributions to describe the feature distribution of multiple samples in a type of attitude capture result, that is, the weighted sum of $Z$ Gaussian distribution functions to approximate the distribution function of each type of attitude observation [31,32].

Suppose that the athlete's typical motion posture is $\partial_{z}$; after classification, it contains a total of $M$ posture samples; namely, $S=\left\{s_{1}, s_{2}, \ldots, s_{M}\right\}$. For a single sample $s_{i}$, the density function of the Gaussian mixture distribution is

$$
\mu(z)=\frac{1}{S_{i}} \sum_{i, j=1}^{M} f_{i j}(x, y) .
$$

Among them, $f_{i j}$ represents the mixing coefficient, that is, the weight, which satisfies

$$
\sum_{i, j=1}^{Z} f_{i j}=1
$$

Among them, $Z=\left\{z_{1}, z_{2}, \ldots, z_{n}\right\}$. In this way, the athlete's motion posture can be described by a submodel containing $Z$ Gaussian distribution functions.
In fact, the result of the activity detection in the previous area will affect the number of sub-Gaussian models and the mean and variance of each submodel. The number of Gaussian models can be estimated using Akaike information criterion and Bayes information criterion, or can be selected based on experience; that is, when the number of samples contained in a typical pose category is small (less than 20 samples), the value of $Z$ is general choose 1 to 2 ; otherwise, when the number of samples is too small, the estimation of the mean and variance in the sub-Gaussian model will be out of generality $[33,34]$.

The calculation of the mean and variance in the subGaussian model is actually a parameter estimation problem in a small sample space. There are many methods for parameter estimation. In comparison, EM is a regression analysis algorithm for incomplete data sets based on the principle of Maximum Likelihood Estimator (MLE). When the data is incomplete, the iterative algorithm for solving the maximum likelihood estimation of the parameters of the distribution density function, which greatly reduces the computational complexity of the maximum likelihood estimation, is conducive to improving the accuracy of the athlete's motion information capture [35, 36].

\section{System Performance Test Experiment}

In order to verify the effectiveness of the designed athlete space-time running information capture system based on virtual reality, the system designed in this paper is used to verify the capture effect of runner running information, and the human knee motion capture system based on MATLAB and the human motion trajectory tracking system based on three frame difference method are compared with the system designed in this paper.

4.1. Experimental Conditions Setting. According to the human skeleton model established above, it was finally decided to select 5 representative running actions for recognition, as shown in Table 3.

A capture experiment was performed on the movement information generated in the five running states shown in Table 3. In the experiment, MATLAB software was used for data processing to generate simulation experiment 
TABLE 3: Running state classification table.

\begin{tabular}{lc}
\hline Serial number & Sports posture \\
\hline 1 & Start \\
2 & Upper limb swing \\
3 & Leg swing \\
4 & Stable trunk \\
5 & Hit the line \\
\hline
\end{tabular}

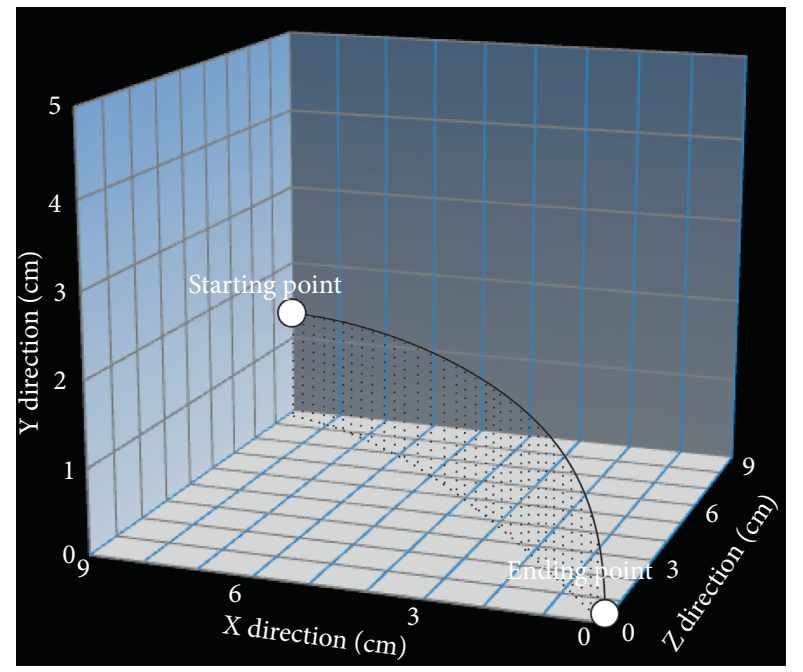

Actual trajectory

(a)

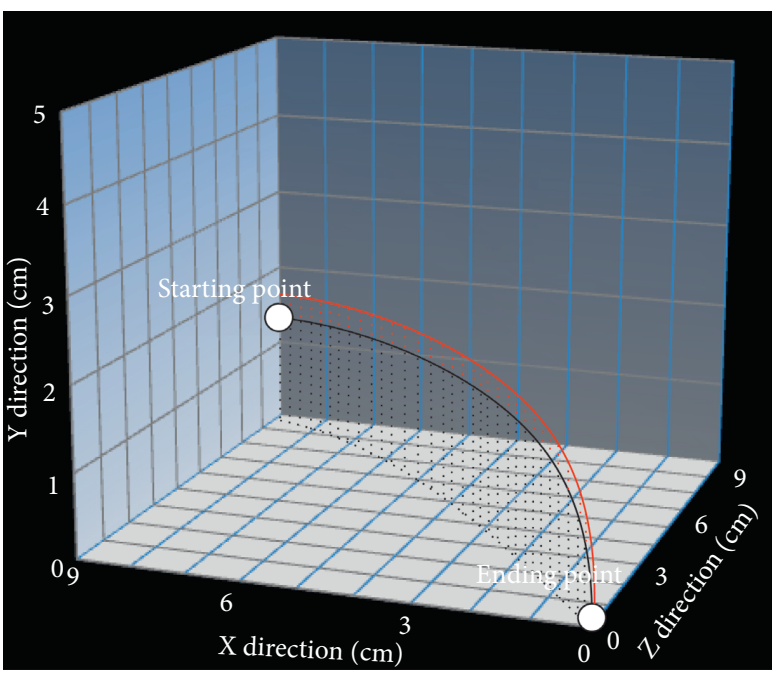

Information capture results Actual trajectory

(c)

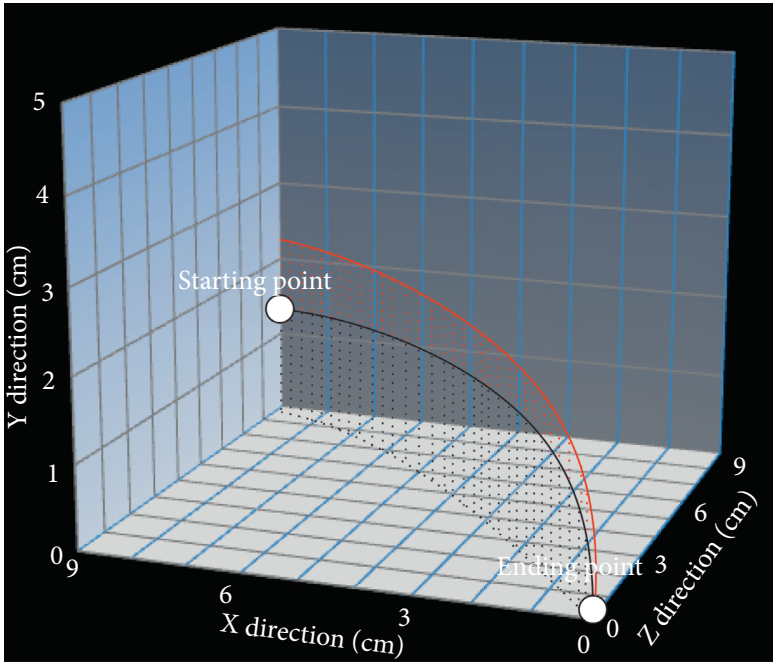

Information capture results Actual trajectory

(b)

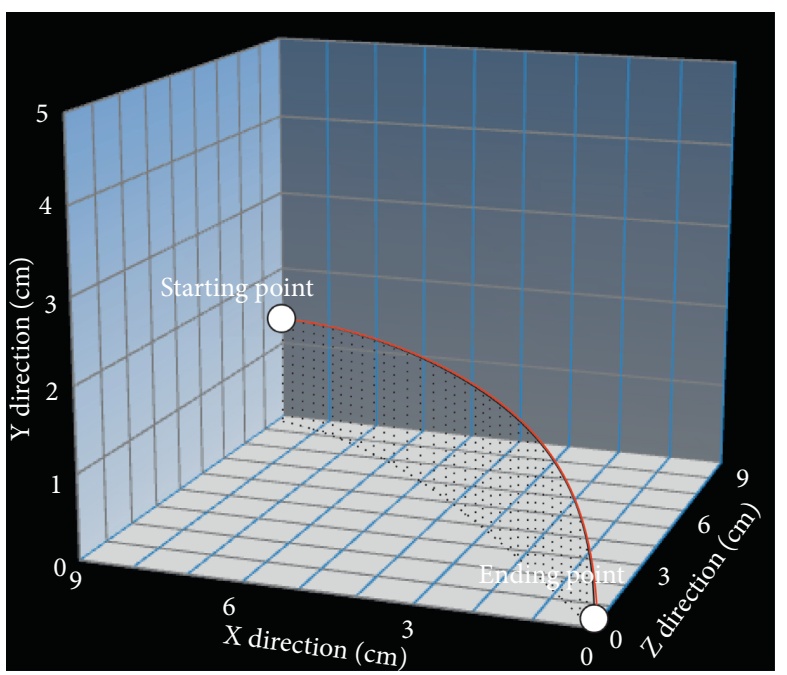

Information capture results Actual trajectory

(d)

FIGURE 6: Comparison results of the accuracy of joint position information capture. 


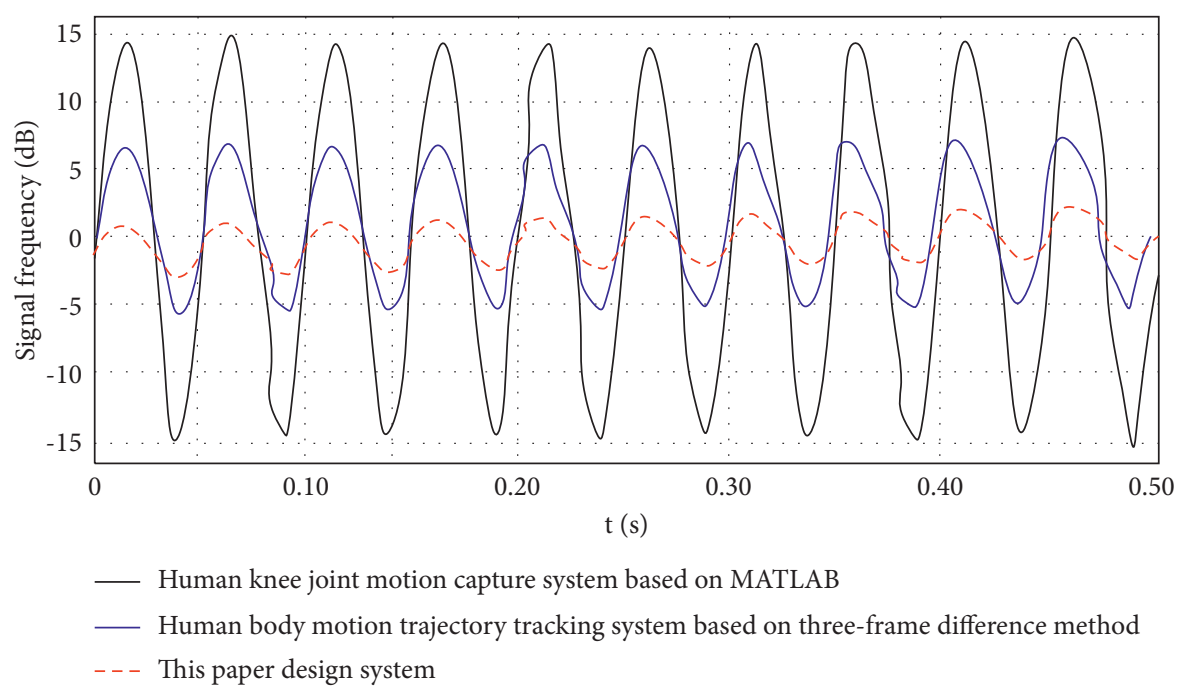

Figure 7: Comparison results of information capture signal components.

diagrams, and related conclusions were drawn based on the analysis of the experimental results.

4.2. Analysis of Experimental Results. Based on the abovementioned experimental environment, a motion information capture experiment is carried out, and the application performance of the system is displayed through three indicators: the accuracy of joint position information capture, the effect of human motion signal processing, and the system processing time.

\subsubsection{Accuracy of Capturing Joint Position Information.} When the athlete is running, the ankle joint is taken as the target, and the accuracy of capturing ankle joint motion information by different systems is analyzed. The result is shown in Figure 6.

According to Figure 6, when three systems are used to capture the motion information of the ankle, the accuracy of information capture shows inconsistent characteristics. Among them, the information capture results of the human knee motion capture system based on MATLAB are obviously different from the actual motion trajectory of the ankle, indicating that the accuracy of information capture is low. Although the information capture effect of human knee motion capture system based on MATLAB is better than that of human knee motion capture system based on MATLAB, there is still a certain gap between its capture results and the system designed in this paper. The coincidence between the information capture results of the lower ankle joint and the actual motion trajectory of the system designed in this paper is high, which shows that the information capture accuracy of the system is high and can provide a reliable data basis for athletes' training. Because the system design in this paper filters the athlete's motion signal and uses the motion information capture algorithm based on the Gaussian mixture model to capture the information, the accuracy of the captured information is improved.
4.2.2. Human Motion Signal Processing Effect. Carrying out the comparison of human motion signal processing effects, the results of information capturing signal components of different systems are shown in Figure 7.

Analyzing Figure 7 shows that in the $0.5 \mathrm{~s}$ capture time, the signal frequency of human knee joint motion capture system based on MATLAB is between $14 \mathrm{~dB}$ and $15 \mathrm{~dB}$. The signal frequency of human body motion trajectory tracking system based on three-frame difference method is between $5 \mathrm{~dB}$ and $7 \mathrm{~dB}$. The signal frequency of this paper design system is between $1 \mathrm{~dB}$ and $3 \mathrm{~dB}$. When the system designed in this paper is used to capture athletes' running information in time and space, the signal output has good stability and a small range of fluctuations up and down, while the signal fluctuation range of the traditional system is large, indicating that the system designed in this paper has better anti-interference ability. This is because the system designed in this paper uses GFSK with strong anti-interference ability for modulation and demodulation in the hardware design, which not only saves energy, but also improves antiinterference.

4.2.3. System Processing Time. In order to further verify the effectiveness of the system designed in this paper, the application performance of different systems is further compared with the system processing time as an experimental indicator. The results are shown in Figure 8.

Analyzing Figure 8 shows that when different systems are used to capture motion information, the processing time of the system has shown a rapid increase in the initial stage of the experiment. As the experiment progresses, the area gradually stabilizes. The comparison shows that the processing time of the system designed in this paper is significantly lower than that of the two traditional systems, which shows that the processing time of the system designed in this paper is shorter and the processing efficiency is higher. It can realize real-time capture of athletes' running information in time and space. Because the system 


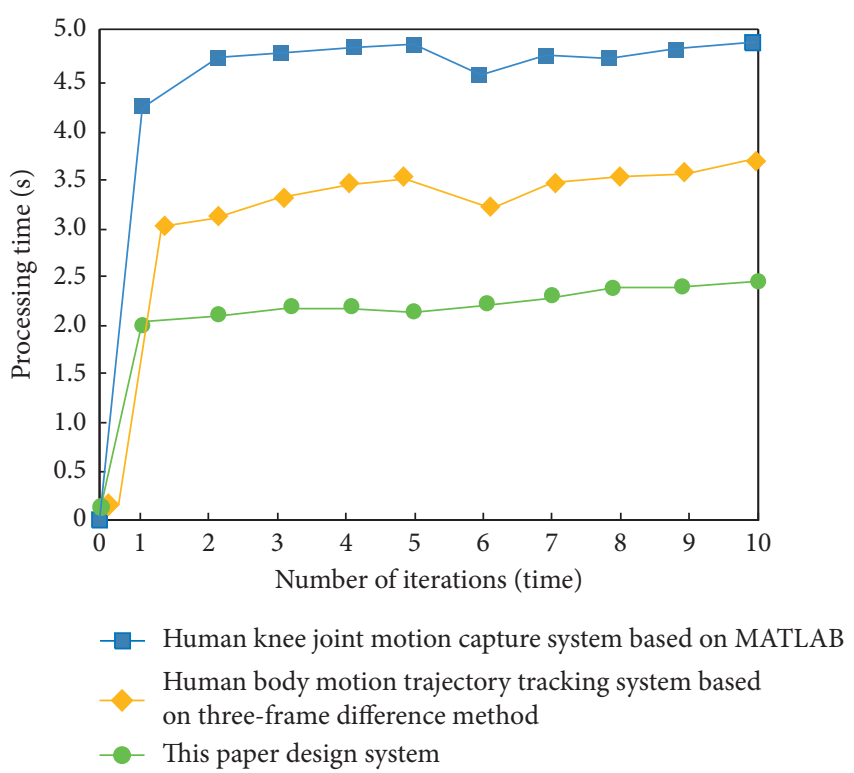

Figure 8: System processing time comparison results.

designed the movement data acquisition module and the data transmission module in this paper, the processing time can be shortened and the processing efficiency can be improved.

\section{Conclusion}

This paper designs an athlete's time-space running information capture system based on virtual reality. In the process of modern sports training, the information capture system based on virtual reality technology is innovative. It can help athletes better master relevant knowledge and technical essentials and greatly improve the training efficiency of athletes. The experimental results show that the system designed in this paper has better capture effect, can achieve accurate and real-time acquisition of athletes' sports information, and has better application effect. In future research, wireless transmission may be adopted. The sensor is attached to the human body, and the data is directly transmitted to the PC for motion tracking through the motion of the human body so as to realize the automatic collection of motion information. The fidelity of the human body model needs to be improved. The pure joints and bones lack a sense of image. In the future, real human models could be made, including skin and hair, which can track and simulate people's subtle movements and even smiles.

\section{Data Availability}

The raw data supporting the conclusions of this article will be made available by the authors, without undue reservation.

\section{Conflicts of Interest}

The authors declare that they have no conflicts of interest regarding this work.

\section{References}

[1] M. Jiang, "Research on athlete training behavior based on improved support vector algorithm and target image detection," Journal of Intelligent and Fuzzy Systems, vol. 39, no. 4, pp. 5725-5736, 2020.

[2] Y. Wang, "Real-time collection method of athletes' abnormal training data based on machine learning," Mobile Information Systems, vol. 2021, no. 3, 11 pages, Article ID 9938605, 2021.

[3] C. Li and J. Cui, "Intelligent sports training system based on artificial intelligence and big data," Mobile Information Systems, vol. 2021, no. 1, 11 pages, Article ID 9929650, 2021.

[4] S. Zhao, "Research on scientific sports training of students majoring in physical education," Revista Brasileira de Medicina do Esporte, vol. 27, no. 5, pp. 460-463, 2021.

[5] Y. Liu and Y. Ji, "Target recognition of sport athletes based on deep learning and convolutional neural network," Journal of Intelligent and Fuzzy Systems, vol. 40, no. 2, pp. 2253-2263, 2021.

[6] L. Tang, C. Zhu, and H. Luo, "Training prediction and athlete heart rate measurement based on multi-channel PPG signal and SVM algorithm," Journal of Intelligent and Fuzzy Systems, vol. 40, no. 4, pp. 1-12, 2020.

[7] V. Isheyskiy, E. Martinyskin, S. Smirnov, A. Vasilyev, and K. Knyazev, "Specifics of MWD data collection and verification during formation of training datasets," Minerals, vol. 11 , no. 8 , p. 798, 2021.

[8] J. P. Wang, X. Zhao, H. Hu, L. Y. Wu, D. Guo, and Y. Li, "Motion capture measurement and analysis of human knee joint based on MATLAB," Journal of Henan Polytechnic University(Natural Science), vol. 39, no. 3, pp. 86-93, 2020.

[9] X. J. Lin, "Design of human body underwater motion trajectory tracking system based on three-frame difference method," Modern Electronics Technique, vol. 42, no. 13, pp. 51-55, 2019.

[10] Q. Li, X. D. Wang, and H. Li, "3D human pose tracking approach based on double Kinect sensors," Journal of System Simulation, vol. 32, no. 8, pp. 1446-1454, 2020.

[11] J. Altet, E. Barajas, D. Mateo, A. Billong, and F. Reverter, "BPF-based thermal sensor circuit for on-chip testing of RF circuits," Sensors, vol. 21, no. 3, p. 805, 2021.

[12] H. Gevorgyan, A. Khilo, Y. Ehrlichman, and M. A. Popovi, "Triply resonant coupled-cavity electro-optic modulators for RF to optical signal conversion," Optics Express, vol. 28, no. 1, pp. 788-815, 2020.

[13] F. Adrion, M. Keller, G. B. Bozzolini, and C. Umstatter, "Setup, test and validation of a UHF RFID system for monitoring feeding behaviour of dairy cows," Sensors, vol. 20, no. 7035, p. 1, 2020.

[14] F. K. Matheus, P. M. Frank, F. B. Marcelo, and G. R. C. Thomas, "RFID wireless system for detection of water in the annulus of a flexible pipe," Marine Structures, vol. 72, no. 3, Article ID 102776, 2020.

[15] J. Flores, I. Garmendia, and I. C. Axpe, "Thermal monitoring and control by infrared camera in the manufacture of parts with laser metal deposition," Dyna, vol. 95, no. 1, pp. 360-364, 2020.

[16] C. Bowen, N. Reeve, T. Pettinger, and J. Gurnell, "An evaluation of thermal infrared cameras for surveying hedgehogs in parkland habitats," Mammalia, vol. 84, no. 4, pp. 354-356, 2020.

[17] A. Krtali, M. Baji, T. Ivelja, and I. Racetin, "The AIDSS module for data acquisition in crisis situations and environmental protection," Sensors, vol. 20, no. 5, p. 1267, 2020.

[18] F. Wen, H. Xiang, T. Zhang, Y. Wang, and X. Gao, "Upgrade of the data acquisition and control system of microwave 
reflectometry on the experimental advanced superconducting tokamak," IEEE Transactions on Nuclear ence, vol. 66, no. 99, pp. 1340-1345, 2019.

[19] H. Aljuaid and S. A. Parah, "Secure patient data transfer using information embedding and hyperchaos," Sensors, vol. 21, no. 1, p. 282, 2021.

[20] M. Helwig, S. Zimmer, P. Lucas, A. Winkler, and N. Modler, "Multiphysics investigation of an ultrathin vehicular wireless power transfer module for electric vehicles," Sustainability, vol. 13, no. 17, p. 9785, 2021.

[21] T. Zhu and F. Zhang, "Design of marine two-way voice communication system based on human-computer interaction," Journal of Coastal Research, vol. 95, no. 1, p. 1389, 2020.

[22] Y. Jing, "Research on fuzzy English automatic recognition and human-computer interaction based on machine learning," Journal of Intelligent and Fuzzy Systems, vol. 39, no. 4, pp. 5809-5819, 2020.

[23] M. Tu, "Gesture detection and recognition based on pyramid frequency feature fusion module and multiscale Attention in human-computer interaction," Mathematical Problems in Engineering, vol. 2021, no. 7, 10 pages, Article ID 6043152, 2021.

[24] G. S. Heidner, P. M. Rider, J. C. Mizelle, C. M. O’Connell, and Z. J. Domire, "Anterior-posterior balance perturbation protocol using lifelike virtual reality environment," Journal of Applied Biomechanics, vol. 36, no. 4, pp. 1-5, 2020.

[25] M. Yamada, K. A. Nikita, J. A. Diekfuss, and L. D. Raisbeck, "The effect of attentional focus on movement accuracy in an immersive and interactive virtual reality environment," Neuroscience Letters, vol. 752, no. 1, Article ID 135814, 2021.

[26] S. Zhang, H. Yu, T. Wang, and J. Dong, "Augmented visual feature modeling for matching in low-visibility based on cycle-labeling of Superpixel Flow," Knowledge-Based Systems, vol. 195, no. 3, Article ID 105699, 2020.

[27] H. Hu, M. Cheng, F. Gao, Y. Sheng, and R. Zheng, "Driver's preview modeling based on visual characteristics through actual vehicle tests," Sensors, vol. 20, no. 21, p. 6237, 2020.

[28] X. Cheng, G. Li, R. Skulstad et al., "Data-driven uncertainty and sensitivity analysis for ship motion modeling in offshore operations," Ocean Engineering, vol. 179, no. 1, pp. 261-272, 2019.

[29] J. Y. Zhao and D. F. Zhang, "Simulation of human motion information capture in time-space domain based on virtual reality," Computer Simulation, vol. 38, no. 8, pp. 391-395, 2021.

[30] L. Li and Q. H. Zhuang, "Prediction and simulation of human behavior continuity based on time domain segmentation," Computer Simulation, vol. 38, no. 5, pp. 339-343, 2021.

[31] I. Shahin and A. B. Nassif, "Novel cascaded Gaussian mixture model-deep neural network classifier for speaker identification in emotional talking environments," Neural Computing \& Applications, vol. 32, no. 7, pp. 2575-2587, 2020.

[32] C. Ananth and D. Brabin, "Enhancing segmentation approaches from Gaussian mixture model and expected maximization to super pixel division algorithm," Sylwan, vol. 164, no. 4, pp. 15-32, 2020.

[33] Z. Hui, S. Jin, D. Li, Y. Z. Yao, and B. Liu, "Individual tree extraction from terrestrial LiDAR point clouds based on transfer learning and Gaussian mixture model separation," Remote Sensing, vol. 13, no. 2, pp. 1-32, 2021.

[34] Q. Xu, S. Yuan, and T. Huang, "Multi-dimensional uniform initialization Gaussian mixture model for spar crack quantification under uncertainty," Sensors, vol. 21, no. 4, p. 1283, 2021.
[35] S. L. Yoo and J. C. Jeong, "Safe navigation distance between marine routes and aquaculture farms in South Korea using Gaussian mixture model," Sensors, vol. 20, no. 5, p. 1246, 2020.

[36] Y. Wang and M. Wen, "Simulation of tennis match scene classification algorithm based on adaptive Gaussian mixture model parameter estimation," Complexity, vol. 2021, no. 1, 12 pages, Article ID 3563077, 2021. 